\title{
Ein schneller enzymatischer Harnstoff-Farbtest als Endpunkt-Methode mit Probenleerwert
}

\author{
A. Bruckner \\ Städtisches Krankenhaus, Zentrallabor, Frankfurt-Höchst
}

\section{Zusammenfassung:}

Es wird eine neue $\mathrm{pH}$-Indikatormethode zur Harnstoffbestimmung beschrieben. Die Methode zeichnet sich durch gute Präzision und Richtigkeit aus. Der aus 150 Wertepaaren ermittelte Korrelationskoeffizient beträgt mit der DAM-Methode $r=0,9970$ und mit der GLDH-Methode $r=0,9971$. Die Richtigkeitsversuche charakterisieren die Methode als praxisgerecht. Wegen der schnellen Durchführung ist die Methode auch für Notfall-Laboratorien zu empfehlen.

\section{Schlüsselwörter:}

Endpunkt-Harnstoffbestimmung - Präzision und Richtigkeit - Methodenvergleich

\section{Summary:}

A new endpoint $\mathrm{pH}$-Indicator method for the determination of Urea is described. Precision and accuracy of the method are good. A comparative study of the results obtained with the Urease/GLDH method and the new endpoint technique showed a good correlation $(r=0,9971)$. Because of its practicability and rapid performance the method is suitable for emergency-laboratories.

\section{Keywords:}

Endpoint Urea determination - precision and accuracy - comparison

\section{Einleitung}

Eine Harnstoffbestimmung ist bei folgenden Fragestellungen angezeigt: akute oder chronische Nierenerkrankung, Azotämien, Fieber, Traumen, Blutungen, Verbrennungen, Transfusionszwischenfällen, Hungerzuständen, Zustände mit gesteigertem Katabolismus bei Kindern, $\mathrm{Di}$ arrhoe und Erbrechen-(1,2). Deshalb ist eine schnelle und billige Harnstoffbestimmung neben der Bestimmung von Kreatinin notwendig $(3,4)$. Nach der vor kurzem im Handel erschienenen Indikator-Methode für die Harnstoffbestimmung stellten wir uns zur Aufgabe, diese Methode als schnelle Endpunktvariante auf das Gerät RA1000 (Technicon ${ }^{\circledR}$ ), das bei uns als Notfallgerät eingesetzt wird, zu adaptieren. In unserer Arbeit vergleichen wir die Diacetylmonoxim - und die vollenzymatische GLDH Methode mit der von uns auf das Gerät RA-1000 adaptierten pH-Indikator-Methode hinsichtlich Präzision, Richtigkeit, Wiederfindung, Linearität sowie Störanfälligkeit gegenüber exogenen und endogenen Substanzen.

\section{Material und Methoden}

\section{Prinzip}

Harnstoff wird durch Urease zu Ammoniumbicarbonat und Ammoniumhydroxid gespalten, wodurch der $\mathrm{pH}$ Wert der Reaktionslösung proportional zu der Harnstoffkonzentration erhöht wird.
In Gegenwart eines $\mathrm{pH}$-Indikators führt die $\mathrm{pH}$-Werterhöhung zu einer proportionalen Extinktionsänderung, die spektralphotometrisch gemessen wird. Voraussetzung für die Proportionalität ist, daß $\mathrm{pH}$-Indikator und Puffer die gleichen pK-Werte haben $(5,14)$.

\section{Reagenzien und Kontrollseren}

Enzymatischer Farbtest „Harnstoff-Duo" und Kontrollserum Duotrol der Fa. Biomed. Harnstoff-Stickstoff-Diacetylmonoxim-Methode, Harnstoff-Endpunkt-Methode mit Urease/GLDH und Calibrator von der Fa. Technicon. Precinorm UPX wurde von Boehringer Mannheim, Validate A von Gödecke AG, Monitrol I von Merz und Dade, Kontrollogen $L$ von Behring bezogen.

\section{Lösungen}

Die Arbeitslösungen für DAM- und Urease/GLDH-Methoden wurden nach Herstellerangaben vorbereitet. Für die $\mathrm{pH}$-Indikator-Methode ${ }^{1}$ wählten wir folgendes $\mathrm{Mi}$ schungsverhältnis:

Reagenz: zu $50 \mathrm{ml}$ Lösung A (Puffer-Indikator-System) gibt man $2,0 \mathrm{ml}$ Lösung $B$ (Urease Suspension), Leerwert-Reagenz: $50 \mathrm{ml}$ Lösung $A$ mischt man mit $2.0 \mathrm{ml}$

\footnotetext{
Aus patentrechtlichen Gründen gibt der Hersteller die Konzentrationsangaben nicht frei
}

Lab.med. 9: 315 (1985) 
$0.9 \%$ iger $\mathrm{NaCl}$-Lösung. Die so hergestellten Reagenzien sind bei $+4^{\circ} \mathrm{C}$ bis vier Wochen haltbar.

\section{Geräte}

Die Vergleichsuntersuchungen wurden am SMA II-Gerät (Technicon) mit der DAM-Methode und am RA-1000 (Technicon) mit der $\mathrm{pH}$-Indikator-Methode und mit der Uiease/GLDH-Methode durchgeführt.

Die Geräteeinstellung am RA-1000 ist aus Tab. 1 zu entnehmen.

Tab. 1: Geräteeinstellung am RA-1000

\begin{tabular}{lc}
\hline Name & 2 HST \\
Typ & 2 \\
$\%$ Pr.Vol. & 15 \\
Filter Pos. & 5 WL 550 \\
Totz. & 200 \\
LW-Reag. & 70 \\
\% Reag.Vol. & $2 \mathrm{MG} / \mathrm{DL}$ \\
Einh. & 1,0000 \\
Unit Fac. & 0 \\
Dez.Punkt & 0,000 \\
RLW UG & 1,500 \\
RLW OG & 0 \\
Linber UG & 220 \\
Lin.Gr. & $\cdot$ \\
Kal.Faktor & $\cdots$ \\
Std.W. & 20 \\
Norm.B. & 45 \\
Norm.B. + & 1,000 \\
Steig. & 0,0000 \\
Schnittp. & 0,0050 \\
EP LIM & \\
\hline
\end{tabular}

- Wird vom Gerät errechnet

-. Wert eines Kalibrationsserums

\section{Ergebnisse}

\section{Reaktionsverlauf}

Anhand eines Humanserums ist in Abb. 1 die Meßsignalentwicklung ohne und mit Urease gezeigt.

Nach der Serumzugabe erfolgt eine gewisse Extinktionszunahme, die auf Serumfarbe und Ammoniakkonzentration zurückzuführen ist. Nach etwa 1 min stabilisiert sich die Probenleerwertextinktion. Nach dem Start mit Urease beginnt die Harnstoffumsetzung, die bei der hier gewählten Urease-Menge nur langsam ihren Endpunkt (4$6 \mathrm{~min}$ ) erreicht.

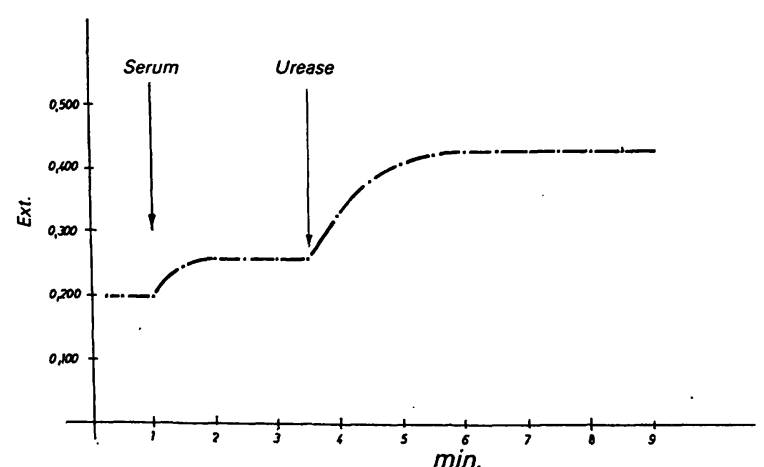

Abb.1: Reaktionsverlauf am RA-1000 der pH-Indikator-Harnstoffbestimmung. Verhältnis Urease: Reagenz $=1: 56$. Messung gegen Luft als Leerwert. Die nach der Serumzugabe meßbare Extinktionsänderung ist auf die endogene Ammoniakkonzentration, Serumfarbe oder Trübung zurückzuführen

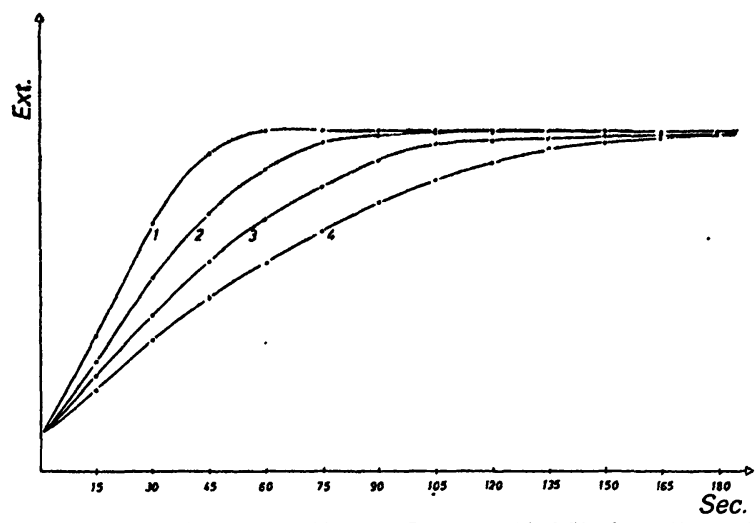

Abb. 2: Optimierung der Urease-Reagenz-Verhältnisse. Kurve 1: Urease: Reagenz = 1:19, Kurve 2: Urease:Reagenz $=1: 26$, Kurve 3: Urease:Reagenz $=1: 36$, Kurve 4: Urease:Reagenz $=1: 51$

\section{Reagenz}

Das Mengenverhältnis Urease zu Reagenz ist davon abhängig, wie schnell der Endpunkt der Reaktion erreicht werden soll.

Die Abb. 2 zeigt den Reaktionsablauf bei einem Harnstoffgehalt des Serums von $33 \mathrm{mmol} / \mathrm{l}$. Daraus ist ersichtlich, daß ein Mischungsverhältnis von 1:26 (Kurve 2) ausreicht, um bei Seren mit erhöhtem Harnstoffgehalt die Reaktion etwa nach 100-120 sec zum Stillstand zu bringen. Bei kleineren Ureaseaktivitäten läuft die Reaktion viel langsamer ab (Kurve 3 und 4). Kurve 1 in Abb. 2 zeigt, daß bei entsprechend hohen Urease-Aktivitäten die Reaktion auch früher ihren Endpunkt erreichen kann. Aus wirtschaftlichen Gründen und aufgrund der im Reagenzpack befindlichen Ureasemenge wählten wir die in der Kurve 2 (Abb.2) gezeigten Verhältnisse. Die so erreichte Urease-Verdünnung mit einem Proben-Reagenzverhältnis von 1:46 (7,5 $\mu$ l Serum und 337,4 $\mu$ l Reagenz) ist laut unseren Messungen ausreichend, die Reaktion bei $37^{\circ} \mathrm{C}$ innerhalb 2 min zum Stillstand zu bringen.

\section{Linearität}

Die Abb. 3 zeigt die Linearität, die mit der beschriebenen Reagenzzusammensetzung erreicht werden konnte. Pro-

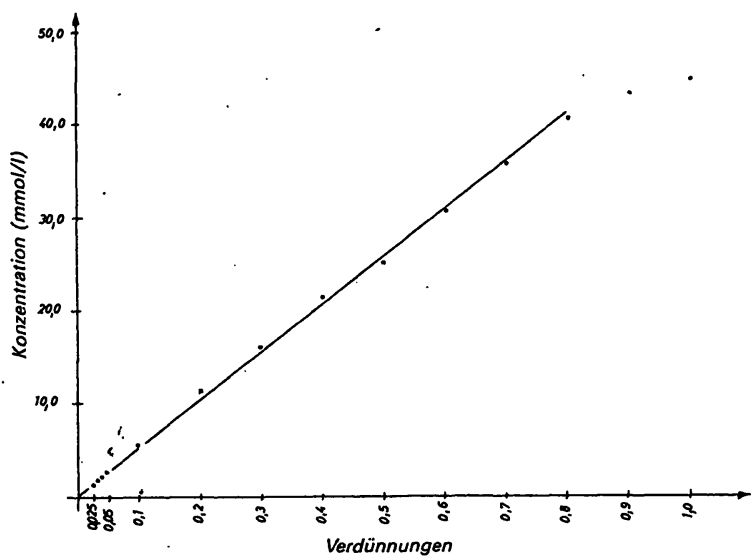

Abb.3: Linearität der $\mathrm{pH}$-Indikator-Harnstoffbestimmung. Verdünnung eines Patientenserums, Mittelwerte aus Doppelbestimmungen 
ben mit einer Harnstoffkonzentration, höher als $37 \mathrm{mmol} / \mathrm{l}$ (=220 mg/dl), müssen verdünnt werden.

\section{Nachweisgrenze}

Die Nachweisgrenze, ermittelt an 24 Tagen, wobei Wasser anstatt Probe analysiert wurde, zeigt einen durchaus akzeptablen Wert von $0,52 \mathrm{mmol} / \mathrm{l}(6,7)$. Somit hat die Methode den sehr guten Meßbereich 0,52-37 mmol/l.

\section{Präzision}

Die Präzision wurde mit verschiedenen Kontroll- und Pool-Seren an mehreren Tagen bestimmt.

Die Tab. 2 zeigt die Präzision in der Serie. Die einzige auffallend schlechte Zahl zeigt unser Pool-Serum mit nieauffallend schlechte Zahl zeigt unser Pool-Serum mit niedrigem Harnstoffgehalt. Diese schlechte Präzision hat sich auch in den Präzisionen von Tag zu Tag bestätigt, was wir mit allgemein schlechteren Präzisionen im unteren

\section{Tab. 2: Präzision der Serie}

\begin{tabular}{llllll}
\hline $\begin{array}{l}\text { Kontroll- } \\
\text { seren } \\
\text { Chargen-Nr. }\end{array}$ & $\begin{array}{l}\text { Soll- } \\
\text { wert } \\
\text { mmol/I }\end{array}$ & n & $\begin{array}{l}\overline{\mathbf{x}} \\
\mathbf{m m o l} / \mathbf{l}\end{array}$ & $\begin{array}{l}\mathbf{S} \\
\mathbf{m m o l} / \mathrm{l}\end{array}$ & $\begin{array}{l}\text { VK } \\
\%\end{array}$ \\
\hline $\begin{array}{l}\text { Duotrol } \\
7280-1\end{array}$ & 7,17 & 21 & 7,5 & 0,1341 & 1,79 \\
\hline $\begin{array}{l}\text { Validate-A } \\
2423091\end{array}$ & 17,99 & 21 & 18,66 & 0,2040 & 1,09 \\
\hline $\begin{array}{l}\text { Moni-Trol I } \\
178\end{array}$ & 6,33 & 21 & 6,70 & 0,1280 & 1,91 \\
\hline $\begin{array}{l}\text { Kontrol- } \\
\text { logen L } \\
623120 \mathrm{~A}\end{array}$ & 8,08 & 21 & 7,99 & 0,1705 & 2,13 \\
\hline $\begin{array}{l}\text { Precinorm } \\
\begin{array}{l}\text { UPX } \\
3-587\end{array}\end{array}$ & - & 21 & 8,88 & 0,0804 & 0,906 \\
\hline Pool $\downarrow$ & - & 21 & 3,99 & 0,2235 & 5,59 \\
\hline Pool $\uparrow$ & - & 21 & 11,89 & 0,1533 & 1,29 \\
\hline
\end{tabular}

Pool $\downarrow=$ Pool-Serum mit niedrigem Harnstoffgehalt

Pool $\hat{\uparrow}=$ Pool-Serum mit erhöhtem Harnstoffgehalt
Bereich auch beim Methodenvergleich bestätigen konnten. Größere Streuungen im unteren Bereich, wie das auch bei Paardifferenzanalysen zu sehen ist, können Fluktuationen der Meßergebnisse verursachen, womit ein höherer VK in der Serie als von Tag zu Tag zu deuten ist.

Den Erwartungen entsprechend zeigt die Präzision von Serie zu Serie, wie auch die von Tag zu Tag, geringfügig schlechtere statistische Zahlen als die in Tab. 1 angegebenen Werte.

Die Präzisionen von Tag zu Tag zeigen einen durchschnittlichen Variationskoeffizienten von $3,4 \%$ (Tab. 3 und 4). Auch die Wiederfindung an Kontrollseren mit einer mittleren Abweichung von - 0,3\% spricht für eine gute Richtigkeit der Methode. Die Wiederfindung ist besser als diejenige, die man mit der Diacetylmonoxim-Methode erreichen kann. Die DAM-Methode liefert in unserer Routine mit den Kontrollserum Monitrol I (Sollwert: $6,16 \mathrm{mmol} / \mathrm{l}$ ) in einem Zeitraum von 14 Monaten eine mittlere Wiederfindung von $-2,8 \%$.

Tab. 3: Präzision von Serie zu Serie

\begin{tabular}{llllll}
\hline $\begin{array}{l}\text { Kontroll- } \\
\text { seren } \\
\text { Chargen-Nr. }\end{array}$ & $\begin{array}{l}\text { Soll- } \\
\text { wert } \\
\mathbf{m m o l} / \mathrm{I}\end{array}$ & $\mathbf{n}$ & $\begin{array}{l}\overline{\mathbf{x}} \\
\mathbf{m m o l} / \mathbf{l}\end{array}$ & $\begin{array}{l}\mathbf{S} \\
\mathbf{m m o l} / \mathrm{I}\end{array}$ & $\begin{array}{l}\text { VK } \\
\%\end{array}$ \\
\hline $\begin{array}{l}\text { Duotrol } \\
7280-1\end{array}$ & 7,17 & 21 & 7,16 & 0,2356 & 3,29 \\
\hline $\begin{array}{l}\text { Validate-A } \\
2423091\end{array}$ & 17,99 & 21 & 19,10 & 0,3468 & 1,82 \\
\hline $\begin{array}{l}\text { Moni-Trol I } \\
178\end{array}$ & 6,33 & 21 & 6,58 & 0,1546 & 2,35 \\
\hline
\end{tabular}

Kontrol-

logen $L$

$\begin{array}{llllll}623120 A & 8,08 & 21 & 8,15 & 0,2552 & 3,13\end{array}$

Precinorm

UPX

\begin{tabular}{llllll}
$3-587$ & - & 21 & 9,08 & 0,2014 & 2,22 \\
\hline Pool $\downarrow$ & - & 21 & 3,99 & 0,1393 & 3,49 \\
\hline Pool $\uparrow$ & - & 21 & 12,24 & 0,3008 & 2,46 \\
\hline
\end{tabular}

Pool $\downarrow=$ Pool-Serum mit niedrigem Harnstoffgehalt Pool $\uparrow=$ Pool-Serum mit erhöhtem Harnstoffgehalt

Tab. 4: Präzision von Tag zu Tag

\begin{tabular}{|c|c|c|c|c|c|c|}
\hline $\begin{array}{l}\text { Kontrollseren } \\
\text { Chargen-Nr. }\end{array}$ & $\begin{array}{l}\text { Sollwert } \\
\mathrm{mmol} / \mathrm{l}\end{array}$ & $\mathbf{n}$ & $\begin{array}{l}\bar{x} \\
\mathrm{mmol} / \mathrm{I}\end{array}$ & $\begin{array}{l}\mathrm{S} \\
\mathrm{mmol} / \mathrm{l}\end{array}$ & $\begin{array}{l}\text { VK } \\
\%\end{array}$ & $\begin{array}{l}\mathbf{R} \\
\%\end{array}$ \\
\hline $\begin{array}{l}\text { Duotrol } \\
7280-1\end{array}$ & 7.17 & 11 & 6,95 & 0,2119 & 3,05 & $-3,07$ \\
\hline $\begin{array}{c}\text { Validate-A } \\
2423091\end{array}$ & 17,99 & 11 & 18,43 & 0,4099 & 2.22 & $+2,44$ \\
\hline $\begin{array}{l}\text { Moni-Trol I } \\
178\end{array}$ & 6,33 & 11 & 6,37 & 0,2988 & 4,69 & $+0,63$ \\
\hline $\begin{array}{l}\text { Kontrollogen L } \\
623120 \mathrm{~A}\end{array}$ & 8,08 & 11 & 7,98 & 0,2732 & 3,42 & $-1,24$ \\
\hline $\begin{array}{l}\text { Precinorm UPX } \\
3-587\end{array}$ & - & 9 & 8,77 & 0.2634 & 3,00 & \\
\hline Pool $\downarrow$ & - & 9 & 3,85 & 0,1943 & 5,05 & \\
\hline Pool $\uparrow$ & - & 9 & 11,88 & 0,3116 & 2,62 & \\
\hline
\end{tabular}

Pool ! = Pool-Serum mit niedrigem Harnstoffgehalt

Pool $i=$ Pool-Serum mit erhöhtem Harnstofigahait 


\section{Richtigkeit}

Die Richtigkeit der Methode wurde durch Regressionsund Korrelationsanalysen sowie durch Paardifferenzana. Iysen geprüft $(8-11)$.

Wie die Abb. 4 zeigt, liefern die beiden Methoden weitgehend übereinstimmende Ergebnisse.

Ahnlich gute Übereinstimmung konnten wir auch gegenüber der Glutamat-Dehydrogenase-Methode erzielen.

Die neue Indikatormethode, die in unserer Adaptation mit Probenleerwert durchgeführt wird, liefert im Durchschnitt 1-2\% niedrigere Werte als die zwei Vergleichsmethoden. Die DAM-Methode liegt im Durchschnitt $0.98 \%$ und die GLDH-Methode 2,0\% höher. Die Ursache für diesen geringfügigen Unterschied ist unserer Meinung nach der mitgeführte Probenleerwert, womit alle Einflüsse von Untergrundfarbe, $\mathrm{pH}$-Wert der Proben und Ammoniakgehalt eliminiert werden. Die Brauchbarkeit der Methode hinsichtlich möglicherweise interferierender Substanzen wie Bilirubin, Hämoglobin und Lipoproteine wurde untersucht. Keine der erwähnten Störmöglichkeiten beeinflußt die Meßergebnisse. Bei Zusatz von Bilirubin bis zu einer Konzentration von $210 \mathrm{mmol} / \mathrm{l}$ und von Hämoglobin bis zu einer Konzentration von $4190 \mathrm{mg} / \mathrm{l}$ zeigt sich keine nennenswerte Interferenz bei der vorgestellten Harnstoffbestimmungs-Methode. Die prozentualen Abweichungen lagen immer innerhalb von $\pm 2 \%$.

Hohe Untergrundfarbe, Trübung sowie erhöhte Ammoniakkonzentrationen werden, wie z. B. bei unseren PoolSeren, durch den Probenleerwert effektiv eliminiert (Abb.6).

Besonders interessierte uns, wie sich das Reagenz bei Seren mit erhöhten Ammoniakkonzentrationen verhält. Unsere Versuche zeigen, daß auch sehr hohe Ammoniak-

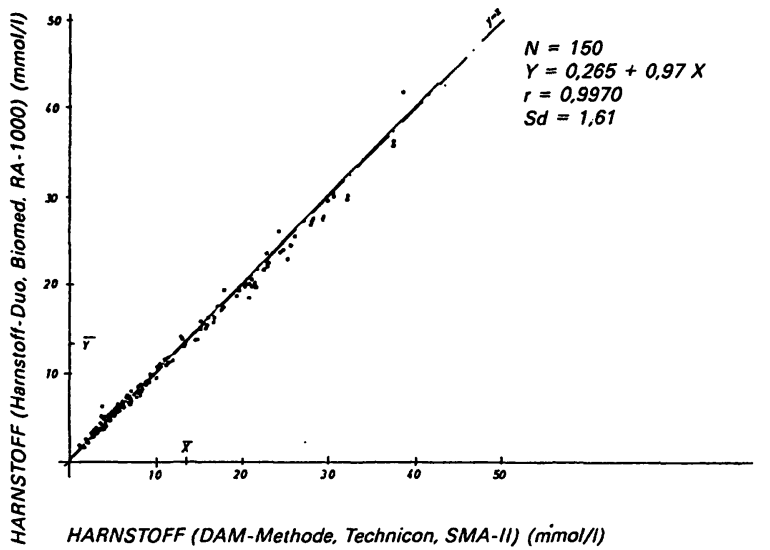

Abb.4: Korrelation und Regression nach der standardisierten Hauptkomponenten-Methode, Verglichen wurde die Diacetylmonoxim-Methode mit der $\mathrm{pH}$-Indikator-Methode

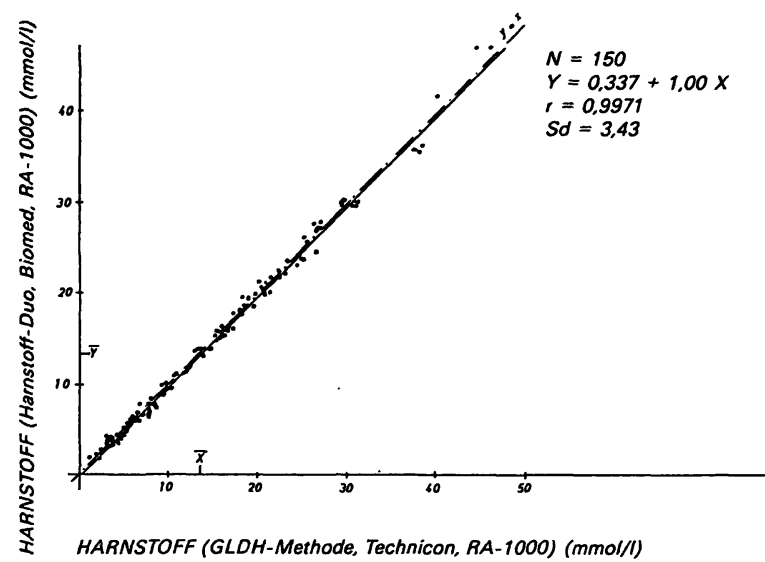

Abb.5: Korrelation und Regression nach der standardisierten Hauptkomponenten-Methode. Verglichen wurde die GLDHMethode mit der pH-Indikator-Methode

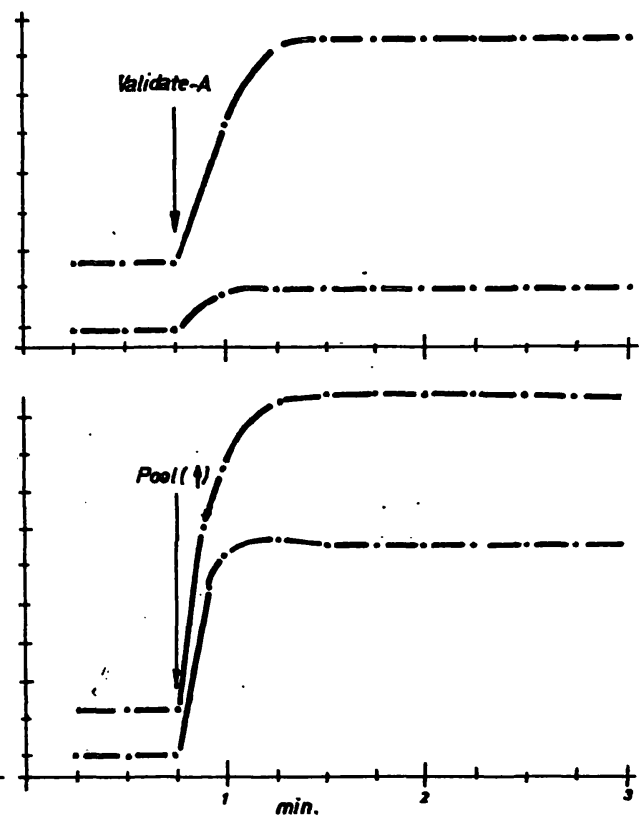

Abb. 6: Einfluß interferierender Substanzen auf die Leerwertextinktion. Die Leerwertextinktionen zweier Kontrollseren (Precinorm UPX und Validate A) sind relativ gering żum Meßwertsignal. Ammoniak- und bilirubinhaltige Proben sowie trübe Proben (unterer Teil der Abbildung) bewirken eine relativ starke Erhöhung des Leerwertsignals zum Meßwertsignal 


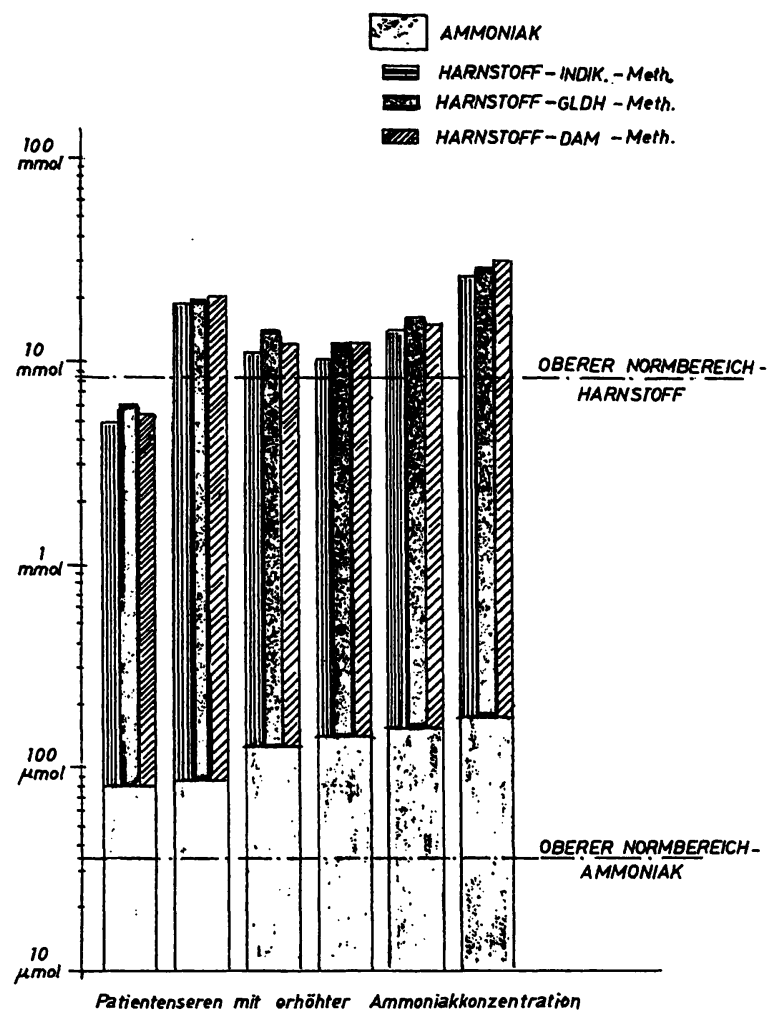

Abb. 7: Harnstoffbestimmungen bei Patientenseren mit erhöhten Ammoniakkonzentrationen

konzentrationen den Reaktionsverlauf nicht stören. Die Ammoniumionen ergeben eine deutlich hohe Probenleerwertextinktion, die am Ende der Reaktion von der Probenextinktion abgezogen wird.

Wie Abb. 7 zeigt, besteht keine meßtechnische Proportionalität zwischen Harnstoff und Ammoniakgehalt. Somit sind auch Plasmaproben, die mit dem Antikoagulans Ammoniumheparinat gewonnen wurden, einsetzbar.

\section{Diskussion}

Die Methode liefert 1-2\% niedrigere Werte im Vergleich zur DAM- und Urease/GLDH-Methode. Damit bestätigt sich die Aussage anderer Autoren (14), die aber vorschlagen, für die neue Indikator-Methode bei der Kalibration die Sollwerte um 3\% höher anzusetzen, um die mit den anderen Methoden ermittelten Sollwerte zu erreichen. Wir sind aber der Meinung, daß die Indikator-Methode in ihrer Endpunktvariante mit Probenleerwert wie auch die Indikator-Methode als kinetische Bestimmung die richtigeren Werte liefert. Die neue Indikator-Methode wird durch Kreatinin, Allantoin, Arginin und Proteine wie das bei der DAM-Methode und durch Ammoniak wie das bei der Urease/GLDH-Methode bekannt ist, nicht gestört $(2,12,13)$.

Zusammenfassend beurteilen wir die neue Indikator-Methode als ein schnelles und billiges (Reagenzkosten pro Analyse DM 0,16), für das Notfall-Labor geeignetes $\mathrm{Be}$ stimmungsverfahren. In dieser von uns erarbeiteten Adaptation liegen die Ergebnisse in 2 min $15 \mathrm{sec}$ vor. Die von Tag zu Tag ermittelten Variationskoeffizienten mit einem Wert von 3,4\% sind gut. Das Reagenz ist lange, mit Urease zusammengemischt, über drei Wochen stabil. Die Korrelation mit anderen Methoden ist überdurchschnittlich gut. Die Methode ist gegenüber vielen Störmöglichkeiten unempfindlich. Es können sogar mit Ammoniumheparinat gewonnene Plasmaproben analysiert werden. Die von uns erarbeitete Endpunktvariante mit Probenleerwert ist mit üblichen Harnstoffbestimmungen vergleichbar.

Danksagung: Der Fa. Biomed danken wir für das Überlassen von Reagenzien.

Schrifttum:

1. PÖGE, A. W., KOTHE, W.: Eine alle Harnstoff(H-N)-Konzentrationen im Serum und Harn erfassende Bestimmungsvariante. Z. ges. inn. Med. 38, 371-374 (1983). 2. THOMAS, L.: Labor und Diagnose, 2. Aufl., S.327-330, Medizinische Verlagsgesellschaft Marburg/Lahn (1984).

3. KRUSE-JARRES, J. D. DUNSBACH, F, GOSSLER, D. KALTWASSER, F. MINDER, W. SASSE, U, BABLOK, W. KOLLER, P U POPPE, W. A.: Harnstoffbestimmung mit Reflotest-Urea in Blut und Serum. Dtsch. med. Wschr. 105, 756-761 (1980).

4. BRUCKNER, A.: Ein neues Reagens für schnelle Harnstoffbestimmung aus $5 \mu \mathrm{l}$ Vollblut, Serum oder Urin. Unveröffentlicht (1974).

5. SKEGGS, L. T. jr.: An automatic method for the determination of carbon dioxide in blood plasma. Amer. J. clin. Path. 33, 181-185 (1960).

6. KAISER, H.: Zum Problem der Nachweisgrenze. Z. Analyt. Chem. 209, 1-18 (1965).

7. STAMM, D.: Recommendations for the description of a selected method in clinical chemistry. J. Clin. Chem. Clin. Biochem. 17. 280-282 (1979).

8. HAECKEL, R.: Statistische Verfahren beim Methodenvergleich. Med. Labor 34, 814 (1981).

9. HAECKEL, R.: 3. Kleinkonferenz über statistische Probleme beim Vergleich klinischchemischer Analysenverfahren. Lab.med. 8. 137-140 (1984).

10. EISENWIENER, H. G., BABLOK, W., BARDORFF, W., BENDER, R., MARKOWERZ, D., PASSING, H., SPAETHE, R., SPECHT, W.: Präzisionsangaben beim Methodenvergleich. Lab.med. 7, 273-281 (1983).

11. SACHS, L.: Angewandte Statistik, 4.Aufl., S.241-249, Springer-Verlag. New York, Heidelberg, Berlin (1974).

12. DIN-NORMENTWÜRFE: Bestimmung von Harnstoff im Serum, Plasma und Harn. Urease-GLDH-Methode (Endpunktverfahren) DIN 58979. Lab.med. 6. A+B 90-92 (1982).

13. BOEHRINGER MANNHEIM GmbH: Testfibel: Harnstoff (1983).

14. HINSCH, W.: Ein neues Prinzip zur Harnstoffbestimmung in der klinisch-chemischen Routineerprobung. Ärtl. Lab. 30, 231-235 (1984).

Anschrift des Verfassers:

Dipl.-Ing. med. Biochem.

Aladár Bruckner

Städtisches Krankenhaus

Zentrallabor

Gotenstraße 6-8

6230 Frankfurt-Höchst 